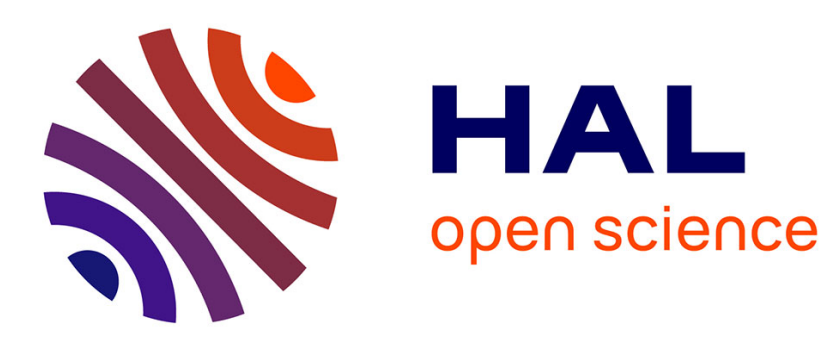

\title{
Spectral nudging in regional climate modelling: How strongly should we nudge?
}

Hiba Omrani, Philippe Drobinski, Thomas Dubos

\section{To cite this version:}

Hiba Omrani, Philippe Drobinski, Thomas Dubos. Spectral nudging in regional climate modelling: How strongly should we nudge?. Quarterly Journal of the Royal Meteorological Society, 2012, 138 (668), pp.1808-1813. 10.1002/qj.1894 . hal-01115488

\author{
HAL Id: hal-01115488 \\ https://hal.science/hal-01115488
}

Submitted on 11 Feb 2015

HAL is a multi-disciplinary open access archive for the deposit and dissemination of scientific research documents, whether they are published or not. The documents may come from teaching and research institutions in France or abroad, or from public or private research centers.
L'archive ouverte pluridisciplinaire HAL, est destinée au dépôt et à la diffusion de documents scientifiques de niveau recherche, publiés ou non, émanant des établissements d'enseignement et de recherche français ou étrangers, des laboratoires publics ou privés. 


\title{
RMetS
}

Royal Meteorological Society

\section{Spectral nudging in regional climate modelling: how strongly should we nudge?}

\author{
Hiba Omrani, ${ }^{\star}$ Philippe Drobinski and Thomas Dubos \\ Institut Pierre Simon Laplace/Laboratoire de Météorologie Dynamique, École Polytechnique/ENS/UPMC/CNRS, \\ Palaiseau, France
}

${ }^{*}$ Correspondence to: H. Omrani, IPSL/LMD, École Polytechnique, F-91128, Palaiseau Cedex, France. E-mail: hiba.omrani@lmd.polytechnique.fr

Spectral nudging is a technique consisting in driving regional climate models (RCMs) on selected spatial scales corresponding to those produced by the driving global circulation model (GCM). This technique prevents large and unrealistic departures between the GCM driving fields and the RCM fields at the GCM spatial scales. Theoretically, the relaxation of the RCM towards the GCM should be infinitely strong provided thre are perfect large-scale fields. In practice, the nudging time is chosen based on trial and error. In this study, the physical parameters setting the optimal nudging coefficient are identified and their effects are discussed. In addition to the predictability time $\tau_{\mathrm{p}}$, already analyzed in a companion article, the time interval $\tau_{\mathrm{a}}$ between consecutive GCM driving fields is a key controlling parameter, especially when spectral nudging is considered. Indeed, the driving GCM fields are interpolated in time at every RCM integration time step, which is much smaller than $\tau_{\mathrm{a}}$. This produces an inaccurate evolution of the GCM fields. A nudging time close to zero (infinitly strong nudging) would thus produce a non-realistic evolution of the RCM large-scale field and consequently an inaccurate small-scale field. The optimum nudging coefficient thus differs from zero, but remains smaller than the predictability time $\tau_{\mathrm{p}}$, as discussed elsewhere. Furthermore depending on the time interval $\tau_{\mathrm{a}}$, all scales present in the driving fields may not be well time-resolved. It can then be beneficial to filter them out rather than driving the RCM with fields affected by time-sampling errors. Copyright (c) 2012 Royal Meteorological Society

Key Words: downscaling; uncertainties; large/small-scale errors; potential vorticity

Received 19 July 2011; Revised 23 November 2011; Accepted 21 December 2011; Published online in Wiley Online Library

Citation: Omrani H, Drobinski P, Dubos T. 2012. Spectral nudging in regional climate modelling: how strongly should we nudge?. Q. J. R. Meteorol. Soc. DOI:10.1002/qj.1894

\section{Introduction}

Dynamical downscaling has been widely used to improve regional climate descriptions at finer scale (e.g. Hewitson and Crane, 1996). It consists in driving a regional climate model (RCM) by large-scale fields provided by a global circulation model (GCM) or (re)analyses as initial and boundary conditions (IC and BC). Previous studies have shown the necessity of relaxing the three-dimensional RCM fields towards the GCM fields to avoid deviation from the large-scale atmospheric circulation (e.g. Alexandru et al., 2007; Lo et al., 2008; Salameh et al., 2010; Omrani et al., 2012). This relaxation technique is also referred to as nudging.

Two different types of nudging exist, both involving ad hoc relaxation times: the spectral nudging which consists of driving the RCM on selected spatial scales only (e.g. Kida et al., 1991; Waldron et al., 1996; von Storch et al., 2000; Raluca Rad et al., 2008) and the indiscriminate nudging which consists in driving the RCM indiscriminately at all scales. Indiscriminate nudging is also referred to as data assimilation, dynamical relaxation, grid-point nudging or analysis nudging (Anthes, 1974; Hoke and Anthes, 1976; 


\section{H. Omrani et al.}

Davies and Turner, 1977; Stauffer et al., 1990; Lo et al., 2008; Salameh et al., 2010; Omrani et al., 2012). For indiscriminate nudging, Omrani et al. (2012) showed that there exists an optimal nudging value $\tau$ closely related to the predictability time $\tau_{\mathrm{p}}\left(\tau \sim 0.4 \tau_{\mathrm{p}}\right)$ which minimizes the error both on the large and small scales.

With indiscriminate nudging, strong nudging is detrimental because it prevents the build-up of small-scale variability. Since spectral nudging does not affect the small scales of the RCM fields, one intuitively expects that the relaxation of the RCM towards the GCM should be infinitely strong provided perfect large-scale fields. However, in the literature, even for spectral nudging, the relaxation time value is not zero (infinitely strong nudging). It is a constant empirically set to produce the most realistic fields (e.g. Raluca Rad et al., 2008).

Thus the question this article addresses is: how strong should we nudge when using spectral nudging technique, and why? To do so, the same technique applied in Omrani et al. (2012) with indiscriminate nudging is used here with spectral nudging. It consists of using the perfect model approach on a nudged quasi-geostrophic model and investigates the physical processes affecting the optimization of the nudging coefficient.

After this introduction, section 2 presents briefly the quasi-geostrophic model and the processing method. Section 3 analyses the quality of the downscaled fields as a function of the nudging time, and discusses the temporal sampling of the driving fields as a function of their spatial scale. Section 4 concludes the study.

\section{The quasi-geostrophic model}

\subsection{Equations}

As in Omrani et al. (2012), we use the flat-bottom two-layer quasi-geostrophic (QG) model on a $\beta$-plane derived by Haidvogel and Held (1980), modifying it only to include the spectral nudging terms. The dimensional form of the equations of motion for such model can be written:

$$
\begin{aligned}
& \partial_{t} Q_{1}+J\left(\Psi_{1}, Q_{1}\right)=-v \nabla^{6} \Psi_{1}, \\
& \partial_{t} Q_{2}+J\left(\Psi_{2}, Q_{2}\right)=-v \nabla^{6} \Psi_{2}-\kappa \nabla^{2} \Psi_{2},
\end{aligned}
$$

where $x$ and $y$ are the zonal and meridional coordinates and where the subscripts 1 and 2 refer to the upper and lower layers of the model, respectively. The quantities $\Psi_{i}$ and $Q_{i}$ are the stream function and potential vorticity (PV) for layer $i, J$ is the horizontal Jacobian operator $J\left(\Psi_{i}, Q_{i}\right)=\left(\partial_{x} \Psi_{i} \partial_{y} Q_{i}-\partial_{y} \Psi_{i} \partial_{x} Q_{i}\right)$ and $\nabla^{2}$ is the horizontal Laplacian operator $\nabla^{2} \Psi_{i}=\Delta \Psi_{i}=\partial_{x}^{2} \Psi_{i}+\partial_{y}^{2} \Psi_{i}$. The two layers have the same depth $H$ at rest. The hyperviscosity $v$ prevents the build-up of enstrophy in high wave numbers and $\kappa$ is a surface friction term. Following Haidvogel and Held (1980), we consider horizontally uniform timeaveraged temperature gradient (directed north-south) and zonal vertical shear. The mean velocity is confined to the upper layer so that $\bar{U}_{2}, \bar{V}_{2}, \bar{V}_{1}=0$ and $\bar{U}_{1}=U$ with $\bar{U}_{i}, \bar{V}_{i}$ the mean zonal and meridional wind components, respectively. Nondimensionalizing $(x, y, t, \psi)$ by $\left(R_{d}, R_{d}\right.$, $\left.R_{d} / U, U R_{d}\right)$ with $R_{d}=\left\{g^{\prime} H /\left(2 f_{0}^{2}\right)\right\}^{1 / 2}$ the Rossby radius ( $g^{\prime}=g \Delta \theta / \theta_{0}$ is the reduced gravity and $f_{0}$ is the Coriolis parameter), the QG PV equations for the transient flow become

$$
\begin{aligned}
& \partial_{t} \widehat{q}_{1}+J\left(\widehat{\psi}_{1}, \widehat{q}_{1}\right)=-\widehat{v} \nabla^{6} \widehat{\psi}_{1}+F_{1}, \\
& \partial_{\hat{t}} \widehat{q}_{2}+J\left(\widehat{\psi}_{2}, \widehat{q}_{2}\right)=-\widehat{v} \nabla^{6} \widehat{\psi}_{2}-\widehat{\kappa} \nabla^{6} \widehat{\psi}_{2}+F_{2} \text {, }
\end{aligned}
$$

where the eddy potential vorticities are:

$$
\begin{aligned}
& \widehat{q}_{1}=\nabla^{2} \widehat{\psi}_{1}+\frac{1}{2}\left(\widehat{\psi}_{2}-\widehat{\psi}_{1}\right), \\
& \widehat{q}_{2}=\nabla^{2} \widehat{\psi}_{2}+\frac{1}{2}\left(\widehat{\psi}_{1}-\widehat{\psi}_{2}\right) .
\end{aligned}
$$

The terms

$$
\begin{aligned}
& F_{1}=-\partial_{\widehat{x}} \widehat{q}_{1}-\left(\widehat{\beta}+\frac{1}{2}\right) \partial_{\widehat{x}} \widehat{\psi}_{1}, \\
& F_{2}=-\left(\widehat{\beta}+\frac{1}{2}\right) \partial_{\widehat{x}} \widehat{\psi}_{2}
\end{aligned}
$$

represent the effects of the mean temperature and planetary vorticity gradients on the transient flow. All variables in Eqs. (5)-(8) are non-dimensional. The parameters which appear in these equations are $\widehat{\beta}=\beta R_{d}^{2} / U, \widehat{\kappa}=\kappa R_{d} / U$ and $\widehat{v}=v /\left(R_{d}^{3} U\right)$. In the following, for sake of simplicity, the hats of non-dimensional variables will be omitted.

As in Omrani et al. (2012), we adopt the 'Big Brother' (BB) experiment approach to drive and evaluate the QG model (Denis et al., 2002). The first step consists in running a highresolution $\mathrm{BB}$ model to produce a high-resolution reference dataset $\left(q_{i}^{\mathrm{ref}}, i=1,2\right)$. Then, the small scales existing in that reference dataset are filtered out to generate a lowresolution dataset $\left(q_{i}^{\text {ana }}, i=1,2\right)$. The filtering technique consists in applying a two-dimensional Fourier filter to $q_{i}^{\text {ref }}$ (subsection 2.2) and the ratio between the horizontal resolutions of $q_{i}^{\text {ref }}$ to $q_{i}^{\text {ana }}$ is hereafter referred to as $\alpha$. The $q_{i}^{\text {ana }}$ fields can be seen as analyses, reanalyses or coarse-resolution GCM outputs. The $q_{i}^{\text {ana }}$ fields are used to initialize and drive another instance of the QG model referred as 'Little Brother' (LB) running at the same resolution and with the same numerical grid as the $\mathrm{BB}$. The $\mathrm{BB}$ reference dataset (before filtering) $q_{i}^{\text {ref }}$ contains the small scales against which the LB small scales are then validated.

\subsection{Nudged version of the QG model}

As discussed in Omrani et al. (2012), if $\psi_{i}^{\text {ana }}$ fields are only used as initial and boundary conditions (absence of nudging), the LB simulated fields $\psi_{i}$ at large scale deviate from $\psi_{i}^{\text {ana }}$ when the integration time is larger than the predictability time $\tau_{\mathrm{p}}$. This is at least true if the numerical domain covered by the LB QG model is sufficiently large (a few Rossby deformation radii), in which case there is no control by the lateral boundary conditions only. In the following, we only consider this situation, which thus requires the use of nudging.

In this article, we use the spectral nudging technique as a natural follow-up of the study by Omrani et al. (2012) on the effect of indiscriminate nudging. With spectral nudging only the large scales are relaxed and Eqs. (3) and (4) become:

$$
\begin{aligned}
\partial_{t} q_{1}+J\left(\psi_{1}, q_{1}\right)= & -v \nabla^{6} \psi_{1}+F_{1}-\frac{1}{\tau}\left(q_{1}^{\text {ls }}-q_{1}^{\text {ana }}\right), \\
\partial_{t} q_{2}+J\left(\psi_{2}, q_{2}\right)= & -v \nabla^{6} \psi_{2}-\kappa \nabla^{6} \psi_{2} \\
& +F_{2}-\frac{1}{\tau}\left(q_{2}^{\text {ls }}-q_{2}^{\text {ana }}\right),
\end{aligned}
$$


where $\tau$ is a freely tunable parameter defined as the nudging time. The shorter the time $\tau$, the closer $q_{i}^{l s}$ and $\psi_{i}^{l_{s}}$ will be to $q_{i}^{a n a}$ and $\psi_{i}^{a n a}(i=1,2)$.

To separate the fine and large scales, we apply a twodimensional Fourier transform of the PV, so that

$$
q(x, y, t)=\sum_{k_{x}, k_{y}} \widetilde{q}\left(k_{x}, k_{y}\right) \exp 2 \mathrm{i} \pi\left(k_{x}+k y\right),
$$

in which $x, k_{x}$ denote zonal coordinates and wavenumbers and $y, k_{y}$ denote meridional coordinates and wavenumbers. The two-dimensional Fourier filter is defined by:

$$
\tilde{q}^{\mathrm{s}}\left(k_{x}, k_{y}\right)= \begin{cases}\tilde{q}\left(k_{x}, k_{y}\right) & \text { if } k_{x}^{2}+k_{y}^{2} \leq k_{\text {cut }}^{2}, \\ 0 & \text { if } k_{x}^{2}+k_{y}^{2}>k_{\text {cut }}^{2},\end{cases}
$$

where $k_{\text {cut }}$ is the cut-off wavenumber. The filtering technique consists in applying this filter to $q_{i}^{\text {ref }}$ so that all scales of $\widetilde{q}_{i}^{l s}$ with a wavenumber higher then $\alpha$ are removed, where $\alpha=k_{\text {cut }} / \max (k)$ is the spectral truncation coefficient. In principle, it is possible to let the nudging coefficient vary with scale, which would make the filter indeed less abrupt than ours. This possibility seems not to be widely used in practice. For instance in Feser and von Storch (2008) the nudging time is chosen to depend on altitude only. In any case our choice of an abrupt filter is motivated by simplicity.

\section{Downscaling using the QG model}

As in Omrani et al. (2012), we set $\widehat{\beta}=0.25, \widehat{\kappa}=0.5$, and $\widehat{v}=0.0001$. The domain size is $24 R_{d} \times 24 R_{d}$ and the number of grid points is $128 \times 128$ (this gives slightly more than 5 points to sample one Rossby deformation radius, which is sufficient as shown in Figure 2 of Omrani et al., 2012). This implies that one Rossby radius is made of 5.3 grid points. The corresponding predictabilty time is $\tau_{\mathrm{p}} \simeq 10$. It has been quantified by computing the initial exponential error growth, yielding the first Lyapunov exponent $\lambda=1 / \tau_{\mathrm{p}}$ (Omrani et al., 2012, provide more details). We run the LB model with different nudging times $\tau$ ranging between $0.01 \tau_{\mathrm{p}}$ and $\tau_{\mathrm{p}}$, and a different spectral truncation coefficients $\alpha=1 / 2,1 / 4,1 / 6,1 / 8$ and $1 / 16$ (not shown).

\subsection{Evaluation methodology}

To quantify the ability of the downscaled LB field $q_{i}$ to reproduce the $\mathrm{BB}$ reference field $q_{i}^{\text {ref }}$ in layer $i$, we first evaluate the variance ratio of $\mathrm{LB}$ to $\mathrm{BB}$ solutions $\sigma_{q_{i}}^{2} / \sigma_{q_{i}^{\text {ref }}}^{2}$, which is a classical diagnostics for climate model evaluation . Using a time interval $\tau_{\mathrm{a}}=\tau_{\mathrm{p}} / 20$, we found a dependance of $\sigma_{q_{i}}^{2} / \sigma_{q_{i}^{\text {ref }}}^{2}$ on $\tau$ similar to the one obtained with indiscriminate nudging (Omrani et al., 2012), with the important exception of the range $0 \leq \tau \leq 0.5 \tau_{\mathrm{p}}$ (not shown). In this range we find $\sigma_{q_{i}}^{2} / \sigma_{q_{i}^{\text {ref }}}^{2} \sim 1$ for both large-scale fields and small-scale fields. With indiscriminate nudging $\sigma_{q_{i}}^{2} / \sigma_{q_{i}^{\text {ref }}}^{2} \sim 1$ only for large scale fields while this ratio computed with small-scale fields increases until it reaches a maximum close to 1 for as $\tau$ goes from 0 to $0.5 \tau_{\mathrm{p}}$. For $0.5 \tau_{\mathrm{p}} \leq \tau \leq 6 \tau_{\mathrm{p}}$, with indiscriminate or spectral nudging, and for both large and small scales, $\sigma_{q_{i}}^{2} / \sigma_{q_{i}}^{2}$ decreases down to a value of about 0.2 then increases up to a value of about 1 . Overall this behavior is consistent with the fact that for small nudging time, the production of small-scale features is inhibited by indiscriminate nudging and not by spectral nudging while for very large values of $\tau$, nudging has no longer any effect, and both small and large-scale fields in LB have the same variance as in BB.

A second approach for LB model evaluation, which corresponds to deterministic evaluation, consists in computing their normalised covariance $a_{i}$ and the correlation coefficient $r_{i}$, defined as

$$
\begin{aligned}
a_{i} & =\frac{\operatorname{cov}\left(q_{i}^{\text {ref }}, q_{i}\right)}{\sigma\left(q_{i}^{\text {ref }}\right)^{2}}, \\
r_{i} & =\frac{\operatorname{cov}\left(q_{i}^{\text {ref }}, q_{i}\right)}{\sigma\left(q_{i}^{\text {ref }}\right) \sigma\left(q_{i}\right)}, \\
\text { where } \quad \sigma\left(q_{i}\right) & =\sqrt{\operatorname{cov}\left(q_{i}, q_{i}\right)}, \\
\text { and } \operatorname{cov}\left(q_{i}^{\text {ref }}, q_{i}\right) & =\overline{\left(q_{i}^{\text {ref }}-\overline{q_{i}^{\text {ref }}}\right)\left(q_{i}-\overline{q_{i}}\right)}
\end{aligned}
$$

where $\bar{q}$ is the spatial average of $q$ (Omrani et al., 2012, provide more details). The quantities $a_{i}$ and $r_{i}$ represent the slope and spread of the scatter plot between $q_{i}^{\text {ref }}$ and $q_{i}$. When $a_{i}$ and $r_{i}$ are close to 1 , the RCM reproduces accurately at each time step and each grid point the reference field. These skill scores are much more constraining than a comparison of climatological statistical diagnostics (Murphy and Epstein, 1989). In order to evaluate quantitatively the quality of the simulations of the fine and large scale features, the LB PV fields $q_{i}$ in the simulations are decomposed into a large-scale part $\left(q_{i}^{l s}\right.$ and $\left.q_{i}^{\text {ref,ls }}\right)$ and a small-scale part $\left(q_{i}^{\text {ss }}\right.$ and $\left.q_{i}^{\text {ref,ss }}\right)$ by application of low-pass and high-pass Fourier filters with cut-off wavelength being the resolution of the field $q_{i}^{\text {ana }}$ driving the simulation.

\subsection{Nudging towards infrequent versus frequent large-scale driving fields}

We now analyse two sets of experiments with $\tau_{\mathrm{a}}=\tau_{\mathrm{p}} / 5$ (infrequent driving fields) and $\tau_{\mathrm{a}}=\tau_{\mathrm{p}} / 20$ (frequent driving fields). First we illustrate qualitatively the effect of the nudging time on the output of the LB. Then for each set of experiment the ability of the LB to reproduce the BB reference fields is evaluated as described in subsection 3.1, as a function of the nudging time $\tau$ and as a function of the spectral truncation coefficient $\alpha$.

Figure 1(a) displays BB large-scale potential vorticity $q_{1}^{\text {ref,ls }}\left(x_{0}, y_{0}, t\right)$ spatially low-pass filtered with spectral truncation coefficient $\alpha$ and sampled at a certain location $\left(x_{0}, y_{0}\right)$ in the domain.

This time series is compared to $q_{1}^{\text {ana }}\left(x_{0}, y_{0}, t\right)$ which is obtained by piecewise linear interpolation of the filtered reference data over intervals of length $\tau_{\mathrm{a}}=\tau_{\mathrm{p}} / 5$. The linear interpolation filters out the variability at short temporal scales, which is of significant amplitude in the reference fields. Figure 1(b) compares $q_{1}^{\text {ref,ls }}\left(x_{0}, y_{0}, t\right)$ and the LB largescale potential vorticity $q_{1}^{\text {ls }}\left(x_{0}, y_{0}, t\right)$ obtained in a strongly nudged simulation $\left(\tau=\tau_{\mathrm{p}} / 10\right)$. The large-scale simulated PV $q_{1}^{\text {ls }}$ is undistinguishable from $q_{1}^{\text {ana }}$, and therefore the high frequency variability of the large-scale field is lost because of the temporal interpolation between two consecutive driving fields $\left(q_{1}^{\text {ana }}\right)$. Figure $1(\mathrm{c})$ finally compares $q_{1}^{\text {ref,ls }}$ 

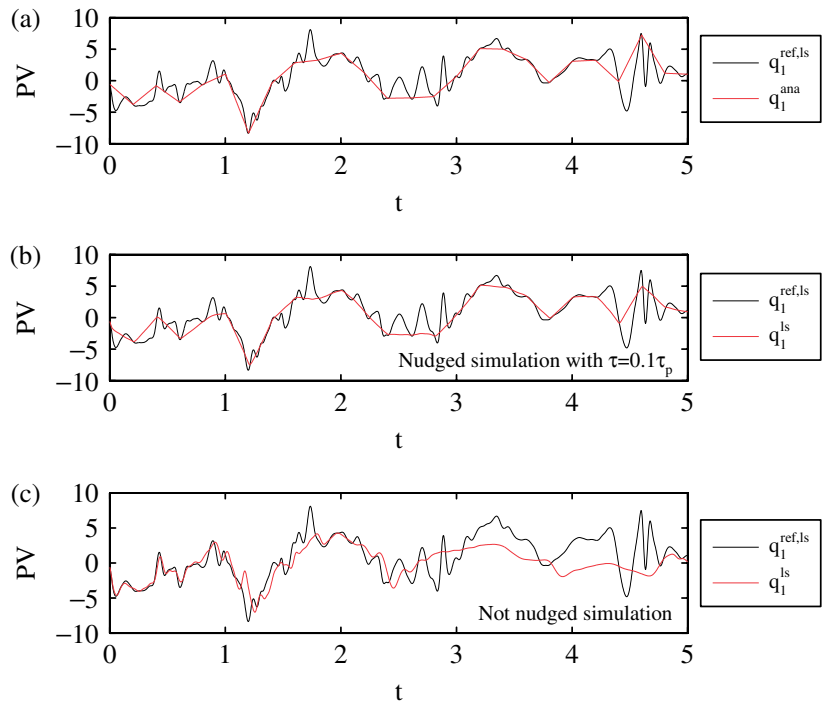

Figure 1. Time evolution of (a) $q_{1}^{\text {ref,ls }}\left(x_{0}, y_{0}, t\right)$ (black) and $q_{1}^{\text {ana }}\left(x_{0}, y_{0}, t\right)$ (red), (b) $q_{1}^{\text {ref,ls }}\left(x_{0}, y_{0}, t\right)$ (black) and $q_{1}^{\text {ls }}\left(x_{0}, y_{0}, t\right)$ (red) for $\tau=0.1 \tau_{\mathrm{p}}$, and of (c) $q_{1}^{\text {ref,ls }}\left(x_{0}, y_{0}, t\right)$ (black) and $q_{1}^{\text {ls }}\left(x_{0}, y_{0}, t\right)$ (red) in the absence of nudging, all with $\alpha=1 / 2$ and $\tau_{\mathrm{a}}=\tau_{\mathrm{p}} / 5$.
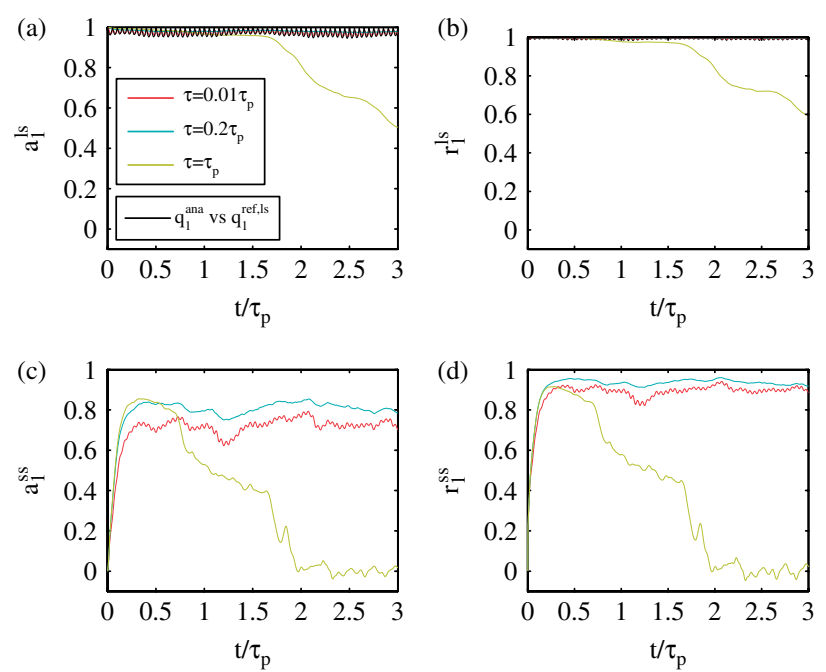

Figure 2. Time evolution of (a,c) covariance coefficient $a_{1}$ and (b, d) correlation coefficient $r_{1}$ computed in layer 1 for the $(\mathrm{a}, \mathrm{b})$ large (ls subscript) and (c, d) small (ss subscript) scales for $\tau_{\mathrm{a}}=\tau_{\mathrm{p}} / 20, \alpha=1 / 2$ and various values of $\tau$.

and $q_{1}^{\text {ls }}$ obtained without nudging. At the beginning of the simulation, there is a near perfect agreement between $q_{1}^{\text {ref,ls }}$ and $q_{1}^{\text {ls }}$. However the two fields start to depart from each other after $t=\tau_{\mathrm{p}}$ and diverge completely for $t>3 \tau_{\mathrm{p}}$. This highlights the necessity of nudging. Is there in between an optimal nudging time that allows the model to create its own large scale dynamics without losing the information present in the driving fields? We will try to answer this question in what follows.

Figure 2 shows the time evolution of the covariance coefficient $a_{1}$ and correlation coefficient $r_{1}$ computed in layer 1 for the small scales (ss subscript) and the large scales (ls subscript) for $\alpha=1 / 2, \tau_{\mathrm{a}}=\tau_{\mathrm{p}} / 20$ and various nudging times .

Between $t=0$ and $0.3 \tau_{\mathrm{p}}$, small scales are produced by the LB model (they are absent from the initial condition at $t=0)$. The black curve in panels $\mathrm{a}$ and $\mathrm{b}$ shows the evolution of $a_{1}^{\text {ls }}$ and $r_{1}^{\text {ls }}$ for $q_{1}^{\text {ana }}$. It displays oscillations which take the value 1 every time $t$ is a multiple of $\tau_{\mathrm{a}}$ (perfect match between $q_{1}^{\text {ana }}$ and $\left.q_{1}^{\text {ref,ls }}\right)$. For a small nudging time (e.g. $\tau=0.01 \tau_{\mathrm{p}}$ ), the LB large-scale field $q_{1}^{\text {ls }}$ is forced to stick to $q_{1}^{\text {ana }}$. This results in similar oscillations of coefficients $a_{1}^{\text {ls }}$ and $r_{1}^{\text {ls }}$ which are close to 1 every time $t$ is a multiple of $\tau_{\mathrm{a}}$, but not as good in between. For the small scales, coefficients $a_{1}^{\text {ss }}$ and $r_{1}^{\text {ss }}$ take fairly high values ( 0.7 and 0.9 , respectively) but display oscillations which indicate unrealistic behaviour of the small-scale dynamics. Coefficients $a_{1}^{\text {ss }}$ and $r_{1}^{\text {ss }}$ evolve in phase with coefficients $a_{1}^{\text {ls }}$ and $r_{1}^{\text {ls }}$, which can be interpreted as the error propagating from the large to the small scales. When the nudging time increases $\left(\tau=\tau_{\mathrm{p}}\right)$, coefficients $a_{1}$ and $r_{1}$, both at large and small scales, tend to low values (about $0.5-0.6$ for large scales due to partial boundary control, and 0 for small scales). The large and the small scales are thus poorly reproduced. An intermediate value of $\tau\left(\tau=0.2 \tau_{\mathrm{p}}\right)$ allows the production of small-scales with good accuracy ( $a_{1}^{\text {ss }}$ and $r_{1}^{\text {ss }}$ equal to 0.8 and 0.95 , respectively) and minimizes the oscillating effect. This advocates for the existence of an optimal nudging time which is different from 0 . When $\tau_{\mathrm{a}}=\tau_{\mathrm{p}} / 20$, the general behaviour is similar but the coefficients $a_{1}^{\text {ss }}$ and $r_{1}^{\text {ss }}$ drop down to 0.4 with much larger oscillations for $\tau$ equal to $0.01 \tau_{\mathrm{p}}$ and $0.2 \tau_{\mathrm{p}}$.

Figure 3 displays the covariance $\left(a_{1}\right)$ and correlation $\left(r_{1}\right)$ coefficients computed in layer 1 for the small (ss subscript) and the large scale (ls subscript) as a function of the nudging time normalised by the predictability time $\left(\tau / \tau_{\mathrm{p}}\right)$ using various resolution factors $\alpha$ and $\tau_{\mathrm{a}}=\tau_{\mathrm{p}} / 20$.

For large scales, coefficients $a_{1}^{\text {ls }}$ and $r_{1}^{\text {ls }}$ decrease as the nudging time $\tau$ increases and as the spectral truncation coefficient $\alpha$ decreases, especially for $\tau \geq \tau_{\mathrm{a}}$. This is in agreement with Omrani et al. (2012). For small scales, coefficients $a_{1}^{\text {ss }}$ and $r_{1}^{\text {ss }}$ exhibit a bell curve for low values of $\tau$ with an optimum for $\tau \simeq 0.2 \tau_{\mathrm{p}}$. Regarding the dependence on $\alpha$, coefficients $a_{1}^{\text {ss }}$ and $r_{1}^{\text {ss }}$ take generally higher values with decreasing $\alpha$ (for $\alpha=1 / 16$ and below, this is no longer true because the Rossby deformation radius is comparable to the grid size). Figure 4 is the same as Figure 3 for $\tau_{\mathrm{a}}=\tau_{\mathrm{p}} / 5$. Similar curves are obtained but especially the coefficients
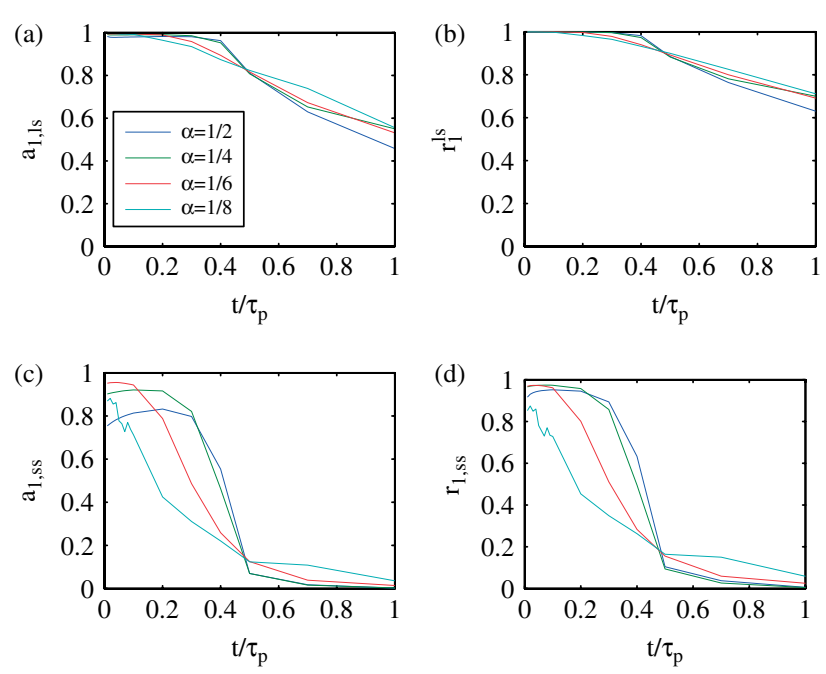

Figure 3. (a, c) Covariance coefficient $a_{1}$ and (b, d) correlation coefficient $r_{1}$ computed in layer 1 for the (a,b) large (ls subscript) and (c,d) small (ss subscript) scales as a function of the nudging time $\tau$ normalised by the predictability time $\tau_{\mathrm{p}}$ using various spectral truncation factors $\alpha$ for $\tau_{\mathrm{a}}=\tau_{\mathrm{p}} / 20$. 

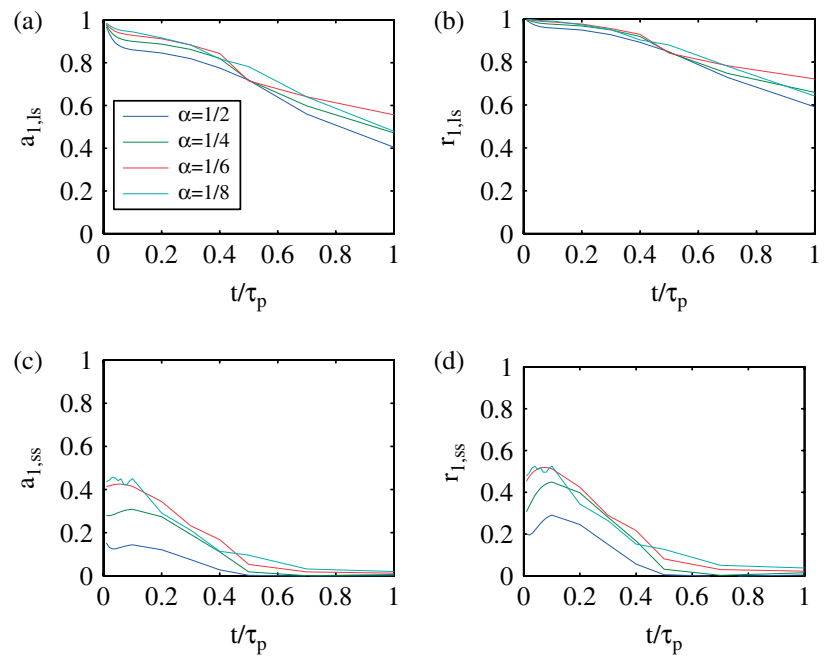

Figure 4. As Figure 3, but for $\tau_{\mathrm{a}}=\tau_{\mathrm{p}} / 5$.

$a_{1, \mathrm{ss}}$ and $r_{1, \mathrm{ss}}$ are much lower than for $\tau_{\mathrm{a}}=\tau_{\mathrm{p}} / 20$ (this is also true for large scales but to a lesser extent).

One can note that in Figure $3\left(\tau_{\mathrm{a}}=\tau_{\mathrm{p}} / 20\right)$, for $\tau<0.2 \tau_{\mathrm{p}}$, coefficients $a_{1}^{\text {ss }}$ and $r_{1}^{\text {ss }}$ are larger for $\alpha=1 / 4$ than for $\alpha=1 / 2$. We now argue that this may be because the 'small scales' present in the large-scale driving fields are not well resolved in time.

\subsection{Relationship between spatial and temporal scales}

To explore this hypothesis, we compute the normalized temporal self-correlation of the reference PV field as a function of the wavenumber and time lag $\tau_{l}$ :

$$
C\left(k_{x}, k_{y} ; \tau_{l}\right)=\frac{\left\langle\widetilde{q}^{*}\left(k_{x}, k_{y} t\right) \tilde{q}\left(k_{x}, k_{y}, t-\tau_{l}\right)\right\rangle}{\left\langle\widetilde{q}^{*}\left(k_{x}, k_{y} t\right) \widetilde{q}\left(k_{x}, k_{y}, t\right)\right\rangle},
$$

where $\langle$.$\rangle denotes temporal averaging.$

Figure 5 displays the normalized self-correlation function for time lag equal to $\tau_{l}=\tau_{\mathrm{p}} / 20$ and $\tau_{l}=\tau_{\mathrm{p}} / 5$ as a function of the wavenumber $k=\left(k_{x}^{2}+k_{y}^{2}\right)^{1 / 2}$. The vertical solid lines indicate the corresponding spectral truncation factors $\alpha$ ranging from $1 / 8$ to $1 / 2$. The two plots start with a moderately high self-correlation near $k=0$ that gradually decrease as a function of wavenumber. This decrease is faster for $\tau_{l}=\tau_{\mathrm{p}} / 5$ than for $\tau_{l}=\tau_{\mathrm{p}} / 20$. This shows, unsurprisingly, that spatial small scales remain self-correlated over short temporal scales. A low self-correlation $C\left(k_{x}, k_{y}, \tau_{l}\right)$ implies that driving large-scale fields sampled at intervals $\tau_{\mathrm{a}}=\tau_{l}$ do not represent accurately the spatial scale $\left(k_{x}, k_{y}\right)$ even if it is nominally present in (resolved by) the large-scale fields. Thus these scales do not represent useful information for the regional model. Injected into the model they can even generate additional errors. It should then be advantageous to let the model generate such scales dynamically itself. This may explain the higher scores obtained with the lowest spectral truncation coefficients.

We now define a critical wave vector $k_{\mathrm{cr}}\left(\tau_{l}\right)$ and a corresponding critical truncation coefficient $\alpha_{\mathrm{cr}}\left(\tau_{l}\right)$ beyond which the small scales are no more correlated. For this we choose $1 / \mathrm{e}$ as a threshold value of the autocorrelation function, i.e. $C\left(k, \tau_{l}\right)<1 /$ e for $k>k_{\mathrm{cr}}\left(\tau_{l}\right)$. For $\tau_{\mathrm{a}}=\tau_{\mathrm{p}} / 20$ (Figure 5(a)), the value of $\alpha_{\text {cr }}$ is around of 0.2 which
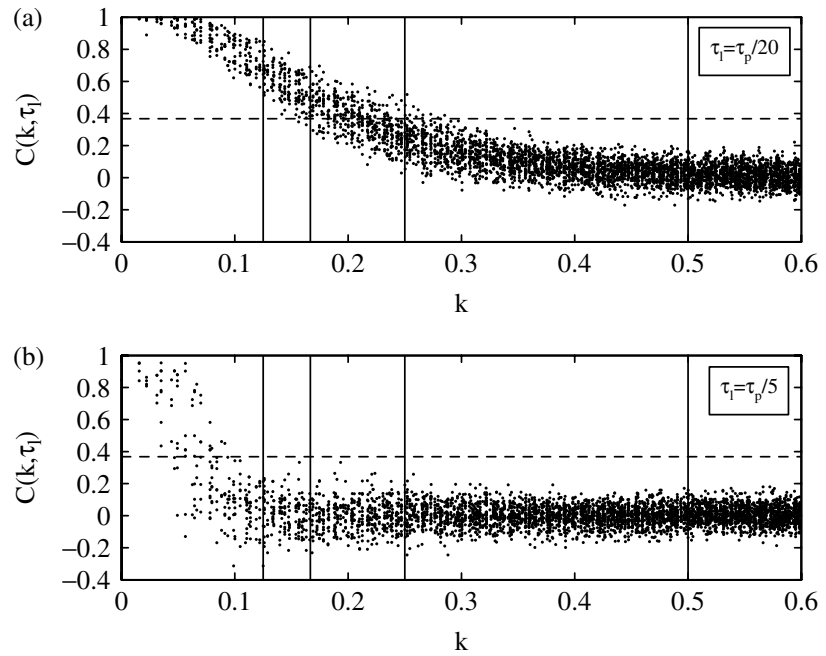

Figure 5. Normalized temporal self-correlation $C\left(k_{x}, k_{y} ; \tau_{l}\right)$ of the reference PV field, as a function of the wavenumber $k=\left(k_{x}^{2}+k_{y}^{2}\right)^{1 / 2}$, for (a) $\tau_{l}=\tau_{\mathrm{p}} / 5$ and (b) $\tau_{l}=\tau_{\mathrm{p}} / 20$.

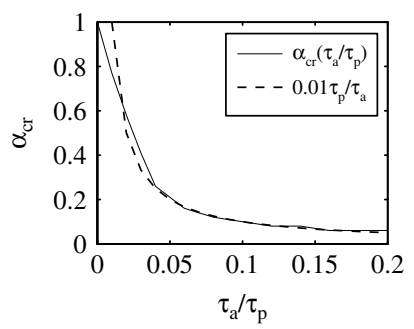

Figure 6. Maximum spectral truncation factor $\alpha_{\mathrm{cr}}$ as a function of the reanalysis interval $\tau_{\mathrm{a}}$.

explains that the small scales corresponding to $\alpha=1 / 4$ are better represented than those for $\alpha=1 / 2$. For $\tau_{\mathrm{a}}=\tau_{\mathrm{p}} / 5$ (Figure 5(b)), scales above $\alpha=0.06$ are not well correlated. Therefore we expect that the highest scores will correspond to $\alpha=1 / 16$ (not shown). However, for this value the model dynamics is not properly solved because the Rossby deformation radius is comparable to the grid size. The highest score correponds to $\alpha=1 / 8$ even if it remains low.

For a given time interval $\tau_{\mathrm{a}}$, if we want to nudge only towards large-scale data that is correctly time-resolved, we should set the spectral coefficient factor $\alpha$ such that $\alpha \leq \alpha_{\mathrm{cr}}\left(\tau_{l}=\tau_{\mathrm{a}}\right)$. Figure 6 summarizes the relationship between the spatial scale of processes and their temporal scale as provided by $\alpha_{\mathrm{cr}}\left(\tau_{\mathrm{a}}\right)$. Except for small $\tau_{\mathrm{a}}$, where all the spatial scales are well correlated in time, $\alpha_{\mathrm{cr}}$ decreases with $\tau_{\mathrm{a}}$, obeying roughly $\alpha_{\mathrm{cr}} \tau_{\mathrm{a}}=$ constant in our idealized set-up. For a large interval, the temporal variability of almost all scales is poorly sampled and $\alpha_{\mathrm{cr}}$ tends to zero.

\section{Summary}

We have analyzed the impact of the time interval of the driving large-scale fields on the outputs of a spectrally nudged model. In Omrani et al. (2012), it has been shown that, for indiscriminate nudging, there is a trade-off between the adverse effect of nudging on small scales and the departure of the large scales from the driving fields. In spectral nudging, this trade-off does not exist since small scales are not affected. Contrary to expectations, an infinitely strong spectral nudging does not produce optimal 


\section{H. Omrani et al.}

reconstruction of the small scales. Indeed, this would be true only if the driving fields were fully resolved in time. However, for practical reasons, the driving large-scale fields are only available every multiple of a certain time interval $\tau_{\mathrm{a}}$, much larger than the model integration step, and are linearly interpolated in time between. This puts a lower bound on the optimal nudging time: there is no gain in reducing the nudging time below the interval $\tau_{\mathrm{a}}$ (the upper bound being a fraction of the predictability time $\tau_{\mathrm{p}}$; Omrani et al., 2012).

Furthermore, there is a relationship between the spatial and temporal variability of the forcing fields. As a consequence, the small scales that have very short characteristic times are poorly sampled in the forcing fields if $\tau_{\mathrm{a}}$ is too long. In this case, the forcing fields are effectively affected by sampling errors. Since $\tau_{\mathrm{a}}$ is usually not a freely adjustable parameter, it is then in fact beneficial to remove the finest and fastest scales from the forcing fields to avoid sampling errors.

Therefore a key factor that limits how strong spectral nudging should be is the finite temporal resolution of the forcing fields. A consequence is that care must be given to their spatial resolution as well to ensure that all the information fed into the model is as correct as possible. The procedure outlined in subsection 3.3 may provide a practical means to check that the forcing fields are adequately timeresolved and to adjust their spatial resolution as necessary. Other driving techniques exist, where the regional model is 'corrected' towards the analyses in an impulsive manner, at regular intervals corresponding to the availability of analyses, and evolves freely in between (Thatcher and McGregor, 2009). The latter technique requires no temporal interpolation but the issue of temporal undersampling remains the same: phenomena occurring at spatial scales resolved by the analyses but at temporal scales unresolved by the available analyses will be erroneously represented and it may be beneficial to filter them out of the data driving the regional climate model.

Of course, the simple nature of the quasi-geostrophic model does not allow us to transpose our results directly to real regional modelling. For instance, the same model and hence the same physics are used in this study. This allows us to isolate the effect of nudging without any interference with other sources of error and uncertainty propagation. In real regional climate modelling, the global climate model used to drive the regional climate model has generally different numerical schemes and physical parametrizations (e.g. Kanamaru and Kanamitsu, 2007; Thatcher and McGregor, 2009). However, the use of dimensionless parameters gives a methodology to evaluate the benefit of spectral nudging with a regional climate model integrating the full complexity of the atmospheric processes. Work in progress gives some confidence in our idealized numerical study, but more thorough analysis is needed to provide a clear picture of the impact of spectral nudging on regional climate modelling with more complex models.

\section{Acknowledgements}

We are grateful to R. Laprise for fruitful discussion and the two referees who helped to improve the manuscript significantly. This research has received funding from the ANR-MEDUP project, GIS 'Climat-Environnement-Sociéte' MORCE-MED project, and through ADEME (Agence de l'Environnement et de la Maitrise de l'Energie) contract $0705 \mathrm{C} 0038$.

\section{References}

Alexandru A, De Elia R, Laprise R. 2007. Internal variability in regional climate downscaling at the seasonal scale. Mon. Weather Rev. 135. 3221-3238.

Anthes R. 1974. Data assimilation and initialization of hurricane prediction models. J. Atmos. Sci. 31: 702-719.

Davies H, Turner R. 1977. Updating prediction models by dynamical relaxation: An examination of the technique. Q. J. R. Meteorol. Soc. 103: $225-245$.

Denis B, Laprise R, Caya D, Côté J. 2002. Downscaling ability of one-way nested regional climate models: The big-brother experiment. Clim. Dyn. 18: 627-646.

Feser F, von Storch H. 2008. A dynamical downscaling case study for typhoons in Southeast Asia using a regional climate model. Mon. Weather Rev. 136: 1806-1815.

Haidvogel DB, Held IM. 1980. Homogeneous quasi-geostrophic turbulence driven by a uniform temperature gradient. J. Atmos. Sci. 37: 2644-2660.

Hewitson BC, Crane RG. 1996. Climate downscaling: techniques and application. Clim. Res. 07: 97-110.

Hoke JE, Anthes R. 1976. The initialization of numerical models by a dynamic initialization technique. Mon. Weather Rev. 104: 1551-1556.

Kanamaru H, Kanamitsu M. 2007. Scale-selective bias correction in a downscaling of global analysis using a regional model. Mon. Weather Rev. 135: 334-350.

Kida H, Koide T, Sasaki H, Chiba M. 1991. A new approach for coupling a limited-area model to a GCM for regional climate simulations. J. Meteorol. Soc. Japan 69: 723-728.

Lo JC, Yang ZL, Pielke RA. 2008. Assessment of three dynamical climate downscaling methods using the Weather Research and Forecasting (WRF) model. J. Geophys. Res. 113(D9): 10.1029/2007JD009 216.

Murphy AH, Epstein E. 1989. Skill scores and correlation coefficients in model verification. Mon. Weather Rev. 117: 572-581.

Omrani H, Drobinski P, Dubos T. 2012. Investigation of indiscriminate nudging and predictability in a nested quasi-geostrophic model. Q. J. R. Meteorol. Soc. 138: 158-169.

Raluca Rad U, Déqué M, Samuel Somo T. 2008. Spectral nudging in a spectral regional climate model. Tellus 60: 898-910.

Salameh T, Drobinski P, Dubos T. 2010. The effect of indiscriminate nudging time on large and small scales in regional climate modelling: Application to the Mediterranean basin. Q. J. R. Meteorol. Soc. 136: $170-182$.

Stauffer D, Seaman N. 1990. Use of four-dimensional data assimilation in a limited-area mesoscale model. Part 1: Experiments with synopticmesoscale data. Mon. Weather Rev. 118: 1250-1277.

Thatcher M, McGregor JL. 2009. Using a scale-selective filter for dynamical downscaling with the conformal cubic atmospheric model. Mon. Weather Rev. 137: 1742-1752.

von Storch H, Langenberg H, Feser F. 2000. A spectral nudging technique for dynamical downscaling purposes. Mon. Weather Rev. 128: $3664-3673$.

Waldron KM, Paegle J, Horel JD. 1996. Sensitivity of a spectrally filtered and nudged limited-area model to outer model options. Mon. Weather Rev. 124: 529-547. 